\section{MS7-P13 Structure and mechanism of reverse transcriptases}

Elzbieta Nowak ${ }^{1}$, Jeniffer T. Miller ${ }^{2}$, Marion K. Bona², Justyna Studnicka ${ }^{1}$, Petr V. konarev ${ }^{3}$, Roman H. Szczepanowski ${ }^{4}$, Wojciech Potrzebowski ${ }^{5}$, Dmitri I. Svergun ${ }^{3}$, Jakub Jurkowski ${ }^{1}$, Janusz Bujnicki ${ }^{5}$, Stuart F. Le Grice ${ }^{2}$, Marcin Nowotny ${ }^{1}$

1. Laboratory of Protein Structure, International Institute of Molecular and Cell Biology, Warsaw, Poland

2. Reverse Transcriptase Biochemistry Section, HIV Drug Resistance Program, Frederick National Laboratory, Frederick, MD, USA

3. European Molecular Biology Laboratory, Hamburg Outstation, Hamburg, Germany

4. Biophysics Core Facility, International Institute of Molecular and Cell Biology, Warsaw, Poland

5. Laboratory of Bioinformatics and Protein Engineering, International Institute of Molecular and Cell Biology, Warsaw, Poland

email: enowak@iimcb.gov.pl

Reverse transcription is a complex process in which single stranded RNA is converted into integration competent double-stranded DNA. This process is exclusively performed by enzyme called reverse transcriptase (RT). The RT-s are a multidomain proteins which consist of $\mathrm{N}$-terminal polymerase domain and a C-terminal RNaseH domain. The multifunctional RTs possess two active sites, first is placed in polymerase domain, where all DNA elongation steps occur and the second is within $\mathrm{RNaseH}$ domain which is responsible for RNA hydrolysis within DNA/RNA hybrid. All of the structural information available for RTs concern the retroviral enzymes $(1,2)$. In contrast there is paucity in equivalent studies on counterpart enzymes of LTR-containing retrotransposons, from which they are evolutionarily derived. We recently solved the first crystal structure of Ty3 RT in complex with its RNA/DNA substrate (3). In contrast to its retroviral counterparts, Ty3 RT adopts an asymmetric homodimeric architecture, whose assembly is substrate-dependent. More strikingly, our structure and biochemical data suggest that the RNase $\mathrm{H}$ and DNA polymerase activities are contributed by individual subunits of the homodimer.

(1) Huang $\mathrm{H}$ et al. "Structure of a covalently trapped catalytic complex of HIV-1 reverse transcriptase: implications for drug resistance" 1998, Science,282, 1669-75.

(2) Nowak E et al. "Structural analysis of monomeric retroviral reverse transcriptase in complex with an RNA/DNA hybrid" 2013, Nucl. Acids Res. 41 3874-87.

(3) Nowak $\mathbf{E}$ et al. "Ty3 reverse transcriptase complexed with an RNA-DNA hybrid shows structural and functional asymmetry" 2014, NSMB, 21 (4) 389-396.

Keywords: reverse transcription, protein DNA/RNA complex
MS7-P14 Viral RNA binding by the human IFIT1-IFIT3 protein complex in the innate immune response.

Maria W. Górna ${ }^{1}$, Gregory I. Vladimer ${ }^{2}$, Yazan M. Abbas ${ }^{3}$, Anna Gebhardt $^{4}$, Matthias Habjan ${ }^{4}$, Beatrice T. Laudenbach ${ }^{4}$, Christina Dimech $^{3}$, Irene Y. Xie ${ }^{3}$, Keiryn L. Bennett ${ }^{2}$, Bhushan Nagar ${ }^{3}$, Andreas Pichlmair ${ }^{4}$, Giulio Superti-Furga ${ }^{2}$

1. Faculty of Chemistry, University of Warsaw, 02-093 Warsaw, Poland

2. CeMM Research Center for Molecular Medicine of the Austrian Academy of Sciences, 1090 Vienna, Austria

3. Department of Biochemistry, and Groupe de Recherche Axé sur la Structure des Protéines, McGill University, Montreal, Quebec H3G 0B1, Canada

4. Innate Immunity Laboratory, Max-Planck Institute of Biochemistry, 82152 Martinsried/Munich, Germany

email: mgorna@chem.uw.edu.pl

The interferon-induced proteins with tetratricopeptide repeats (IFITs) have recently emerged as a potent innate immune effectors that bind non-self RNA, which results in the inhibition of translation of viral transcripts. The structure of IFIT5 reveals the mode of recognition of the 5' triphosphate (PPP) group on RNA, whereas IFIT1 can recognize both 5'-PPP or cap 0 groups. IFIT1 interacts with IFIT3, which has no known RNA binding capability on its own, and for which the role in the larger multi-IFIT complex is elusive. Here, we begin the dissection of the role of the higher-order IFIT complexes and demonstrate that the IFIT1-IFIT3 complex binds RNA with a higher affinity than IFIT1 alone. The IFIT1-IFIT3 interaction, which is mediated by the last tetratricopeptide repeat motifs in both proteins, is necessary for the full antiviral effect of IFIT1 against VSV. In cells, IFIT1 and IFIT3 associated together, and re-distributed and co-localized together with PPP-RNA. We propose a new role for IFIT3 as an enhancer of IFIT1 activity. Regulation of the IFIT1-IFIT3 complex may provide additional possibility for signal integration in the antiviral response.

Keywords: tetratricopeptide repeats, RNA binding, IFIT 